\title{
Analysis of Monitoring System Reliability for Wind Turbine Based on Wireless Sensor Network
}

\author{
Fu Zhixin ${ }^{1}$, Liu Zhen ${ }^{*}, 1$, Ding Guorong ${ }^{1}$, Li Hanbing ${ }^{2}$, Zhao Min $^{1}$, Li Qiaomu $^{1}$ and Yuan Yue ${ }^{1}$ \\ ${ }^{I}$ College of Energy and Electrical Engineering, Hohai University, 8 Focheng West Road, Jiangning District, Nanjing, \\ Jiangsu, 211100, China \\ ${ }^{2}$ State Grid Lianyungang Power Supply Company, 1 Xingfu North Road, Xinpu District, Lianyungang, Jiangsu, 222000, \\ China
}

\begin{abstract}
In this paper, based on wireless sensor network technology, we propose the monitoring system for wind turbine. In order to ensure that the monitoring system can completely and accurately obtain the condition monitoring information, using exponential distribution function, we establish the reliability model. Because the topology control techniques can improve the monitoring system reliability, we present the hierarchical network topology for monitoring system. Then, combined with the presented hierarchical network topology, we study the reliability model. Finally, simulation shows that the network parameters affect the reliability and the working time of monitoring system.
\end{abstract}

Keywords: Wind turbine, monitoring system, reliability, wireless sensor network, network parameters.

\section{INTRODUCTION}

As a kind of clean and renewable energy power generation, wind power can not only effectively solve deterioration problems of ecological environment, but also meet adjustment demands of energy structure. Therefore, wind power has developed rapidly [1]. However, wind turbine is normally located in remote area with harsh environment and climate variability. Moreover, its structure is very complicated. Wind turbine is easy to be damaged, which causes operation faults. Thus, it is very necessary to monitor reliably wind turbine condition.

Traditional regular maintenance and fault maintenance lack the mechanism to grasp operation status of wind turbine. They have drawbacks of high cost and low fault repair rate [2]. The maturer existing monitoring system of wind turbine uses wired monitoring technology having problems of high-cost investment, difficult equipment installation and so on [3]. The condition monitoring system based on wireless sensor network (WSN) is a kind of low-power, low-cost, self-organization, and miniaturization monitoring system. The WSN-based monitoring system of wind turbine (WSNMSWT) can solve problems of wired monitoring and it can provide real time condition monitoring for wind turbine [4].

In recent years, with the capacity increase of wind turbine, it is more difficult to carry out the operation maintenance. As a result, the WSN-MSWT has received widespread attention. Nodes deployment for wind turbine is the first problem to solve [5]. And how to set up the network planning of wind turbine monitoring based on WSN makes sense [6]. Additionally, the monitoring strategy should consider the mechanical transmission system of wind turbine [7]. In [7], authors design the hardware and software of monitoring system, and gives the hardware structure of nodes. Furthermore, in [8], authors propose the approach in sensing wind speed for wind energy using WSN technology. Moreover, according to Spacek [9], monitoring mechanical vibration in wind turbines is very important, but it ignores the real network topology of WSN.

The WSN-MSWT operates normally in bad environment. In order to obtain completely and accurately the condition monitoring information of wind turbine, it is necessary to analyze the monitoring system reliability. We can improve the monitoring system reliability considering the network lifetime [10]. Topology control techniques can extend lifetime of WSN applied is smart grid [11]. But authors do not determine whether topology techniques can influence the monitoring system reliability. Besides, we should take into account the system average working time to evaluate the monitoring system reliability [12]. According to [12], total amount of subsystems and nodes influence the monitoring system reliability.

When the WSN-MSWT runs, the nodes in each cluster will separately collect and process condition information of wind turbine, then upload the information to the cluster head node. The cluster head node can communication with other cluster head nodes, and transmit the monitoring information to base station.

This paper presents the topological structure of the monitoring system for wind turbine. Furthermore, we establish the reliability model and research the monitoring system reliability. Finally, simulation analysis shows the influence of network topology on the monitoring system reliability. 


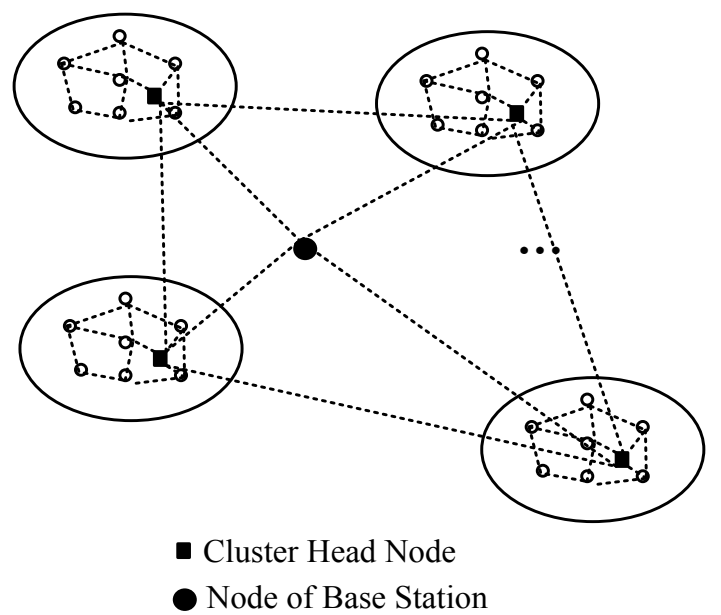

Fig. (1). Hierarchical topology of monitoring system.

\section{NETWORK TOPOLOGICAL STRUCTURE OF MONITORING SYSTEM}

\subsection{Selection of Network Topology}

Topological structure of WSN is divided into three kinds: star, mesh and hybrid type [13, 14]. Star topology has the simple single-hop structure. This topology consumes small energy, but its communication reliability is poor. Mesh topology is complex because of the multi-hop structure. This topology has strong communication robustness while its energy consumption is very high. Hybrid topology is mixture of star topology and mesh topology. It is characterized by its small energy consumption and strong flexibility. Among these topologies, the hierarchical topology is a typical hybrid topological structure. In the hierarchical topology, sensor nodes are divided into several independent clusters, and cluster head node should be selected by a certain algorithm. The cluster head node is responsible for receiving monitoring information from other nodes in the cluster. After being processed, the monitoring information will be sent to base station.

The topological network structure needs to realize independent monitoring for different components of wind turbine, and different components can communicate with each other. Thus, the topology of monitoring system should be hierarchical. Based on above analysis, the topological structure of WSN-MSWT should adopt the mixed network topology to have the hierarchical monitoring (Fig. 1).

The network topology of monitoring system in this paper is the mixture of star topology and mesh topology, wherein each inner subnet uses the mesh network, and network between the subnet and the base station adopts the star network. The monitoring system consists of $m$ subnets, each subnet is composed of $n$ nodes. Each subnet operates individually, uninfluenced by other subnets. Table 1 corresponds to the distribution of subnets in various positions of wind turbine.
Table 1. Distribution of subnets in wind turbine.

\begin{tabular}{|c|c|c|c|}
\hline Position & Blade & TRANSMISSION SYSTEM & Engine room \\
\hline \hline subnets amouts & $m_{1}$ & $m_{2}$ & $m_{3}$ \\
\hline
\end{tabular}

In Table 1, $m=m_{1}+m_{2}+m_{3}$. In actual deployment, values of $\mathrm{m} 1, \mathrm{~m} 2, \mathrm{~m} 3$ and $\mathrm{n}$ are chosen depending on the specific shape of each component in wind turbine.

\subsection{Selection of Monitoring Points}

We should determine the hierarchical network topology of WSN-MSWT depending on specific circumstances of monitoring points in wind turbine.

The working environment of wind turbine is relatively bad, wind turbines easily break down. Among components in wind turbine, the occurring faults in transmission system have the highest percentage. Therefore, under the premise of ensuring the reliability of WSN-MSWT, it is important to study sensor nodes deployment in the transmission system [15]. Vibration parameters are characteristic quantity. They can more accurately reflect operation status of transmission system in wind turbine, they are also easy to be detected. Thus, this paper deploys sensor nodes in monitoring points and in their nearby positions to monitor and to get vibration information of wind turbine. Then, the WSN-MSWT can diagnose the operation condition of wind turbine. The distribution of monitoring points in wind turbine is illustrated in Fig. (2).

We select six monitoring points (Fig. 2): monitoring point (1) is responsible for monitoring spindle bearing condition. Monitoring points (2) and (4) are respectively responsible for the input and output vibration monitoring of the spindle bearing. Monitoring point (3) is responsible for monitoring planetary gear of gearbox. Monitoring point (5) is responsible for monitoring input end bearing of engine. 


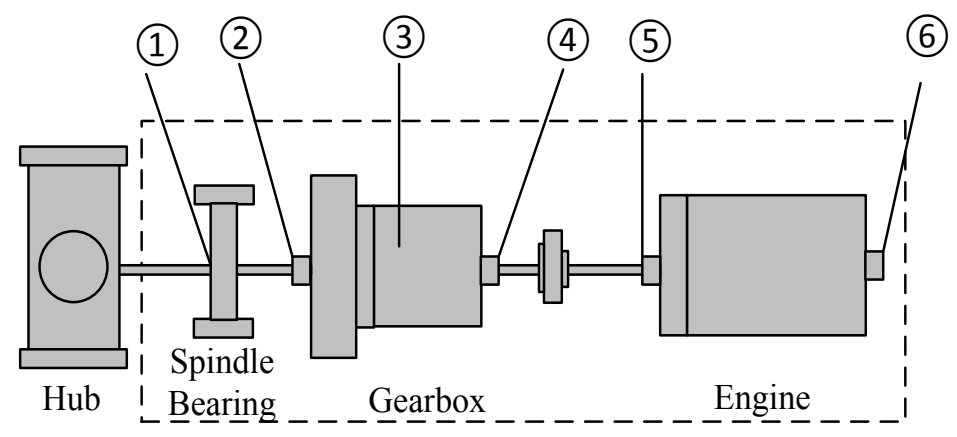

Engine Room

Fig. (2). Distribution of monitoring points in wind turbine.

Monitoring point (6) is responsible for monitoring free end bearing of the engine. In addition to the above six monitoring points, a monitoring point should be put in cabin deck used for vibration condition monitoring of engine room and connected to the tower. Moreover, we should put a monitoring point in blade root that is easy to break down. We use this monitoring point to gain blade vibration information.

Obviously, amount of the monitoring points for transmission system should be larger, and monitoring different positions for transmission system is more important. For the purpose of comprehensive information acquisition of monitoring points for transmission system, and of the reliability of communication between subnets and base station, the transmission system is composed of more than one subnet, namely $m_{2}$ represents a set of subnets. We will discuss below about how to combine monitoring needs of transmission system and how to guarantee the reliability of the constructed subnet systems.

\section{RELIABILITY MODEL OF MONITORING SYSTEM}

\subsection{Concept and Definition of System Reliability}

(1) System Reliability

Reliability is a kind of probability: in the specified conditions and within the stipulated time, the probability of completing prescribed tasks [16]. We usually denote reliability index function as $R(t)$, called as reliability function. The constant $\mathrm{T}$ represents time for research object from normal state to fault state, namely the object lifetime. The reliability function can be expressed as:

$$
R(t)=\mathrm{P}\{\mathrm{T}>t\}
$$

Equation (1) means the probability for an individual or a system of not breaking down between $(0, t)$.

\section{(2) Cumulative Distribution Function}

The cumulative distribution functions refers to the unreliability that means the probability of not completing tasks under specified conditions and within prescribed time period for hydraulic products. The cumulative function $F(t)$ is generally presented by:
$R(t)=\mathrm{P}\{\mathrm{T} \leq t\}$

Because the completed event and uncompleted event within the prescribed time are a pair of opposite event, the following equation can be obtained:

$F(t)=1-R(t)$

Equation (3) describes the probability of fault for an individual or a system between $(0,1)$.

(3) Probability Density Function of Fault

The probability density function of fault $f(t)$ is a derivative of unreliability, namely

$f(t)=\frac{\mathrm{d} F(t)}{\mathrm{d} t}$

(4) Fault Rate

The fault rate $\lambda$ refers to, in any time $t$, the probability of fault occurrence of an individual in a unit of time.

Assuming that the studying individual number is $N$, fault number after $t$ is $r(t)$. Then fault number after $t+\Delta t$ is $\mathrm{r}(t+$ $\Delta t)$, so the fault rate can be expressed by:

$\lambda(t)=\frac{r(t+\Delta t)-r(t)}{[N-r(t)] \cdot \Delta t}$

When $\mathrm{N} \rightarrow \infty$ and $\Delta t \rightarrow 0$, take limit of Equation (5):

$\lambda(t)=\lim _{N \rightarrow \infty} \frac{r(t+\Delta t)-r(t)}{\Delta N \rightarrow 0}=\lim _{N \rightarrow \infty} \frac{\frac{r(t+\Delta t)-r(t)}{N \cdot \Delta t}}{\frac{N-r(t)}{N}}$

The estimation for reliability is shown in Equation (7):

$R(t)=\frac{N-r(t)}{N}$

Expression for the probability density estimation is given in Equation (8):

$f(t)=\frac{r(t+\Delta t)-r(t)}{N \cdot \Delta t}$

Solve equations from (6) to (8):

$\lambda(t)=\frac{f(t)}{R(t)}$ 
From equations (3) and (4), Equation (10) is as follows:

$f(t)=\frac{\mathrm{d} F(t)}{\mathrm{d} t}=-\frac{\mathrm{d} R(t)}{\mathrm{d} t}$

When Equation (10) is substituted into Equation (9), the following equation can be obtained:

$\lambda(t)=-\frac{1}{R(t)} \cdot \frac{\mathrm{d} R(t)}{\mathrm{d} t}$

Take integral for either side of the equals sign in Equation (11):

$\int_{0}^{t} \lambda(t) \mathrm{d} t=-\ln R(t)+\ln R(0)$

In the case of knowing $\lambda(t)$ and $R(t)$, we can solve the normal working time $t$.

\section{(5) Average Normal Working Time (ANWT)}

In the reliability study of an individual or a system, the ANWT is a very important characteristic quantity, we can use it directly to reflect the lifetime of study object. In the experimental study of actual sample, the normal working time from the first sample to the $n_{\text {th }}$ sample is presented as $t_{1}$, $t_{2}, t_{3} \ldots t_{n}$, assuming that there are $n$ samples:

$\mathrm{ANWT}=\frac{1}{n} \sum_{i=1}^{n} t_{i}$

When the ANWT is generalized to an abstract system, it can be presented by the expectation of normal working time of monitoring system:

$$
\mathrm{ANWT}=\mathrm{E}(t)=\int_{0}^{\infty} R(t) \mathrm{d} t
$$

\subsection{Reliability Model of Sensor Nodes}

To describe the system reliability, there are usually models of binomial distribution, exponential distribution, Gamma distribution, Weil-bull distribution, normal distribution and so on. Among them, the exponential distribution is a type of single parameter distribution, and it has good adaptability. Therefore, to establish the reliability model of sensor nodes, we apply the exponential distribution model, namely nodes lifetime is subject to exponential distribution with fault rate $\lambda$. Then the reliability model can be expressed as:

$$
R(t)=1-F(t)=\mathrm{e}^{-\lambda t}, t>0
$$

When Equation (15) is substituted into Equation (14), the following equation can be obtained:

$\mathrm{ANWT}=\int_{0}^{\infty} e^{-\lambda t} \mathrm{~d} t=\frac{1}{\lambda}$

Usually the value of ANWT is taken as $10^{6}$ hours. When it is substituted into Equation (16), the reliability function of sensor nodes in the monitoring system for wind turbine is:

$R_{0}(t)=\mathrm{e}^{-10^{-6} t}, t>0$

\subsection{Reliability Model of Monitoring System}

We can decide the reliability of the WSN-MSWT by the reliability of each subnet of monitoring system. Considering impact of each subnet on the reliability of the whole monitoring system, the system can be divided into series system, parallel system, hybrid system and voting system. Among these four kinds of systems, in the series system, if fault occurs in any subnet system, the whole system will malfunction. In the parallel system, if fault occurs in all subnet systems, the whole system will malfunction. Hybrid system is a mixture of series system and parallel system. In the voting system, as long as the number of subnet systems is not less than a certain constant, the whole system will not have faults. Each subnet system in the WSN-MSWT works alone. Therefore, it necessary to study reliability of independent subnet systems before studying reliability evaluation model of whole monitoring system of wind turbine.

This paper uses mesh structure for each subnet system. For a mesh network containing $n$ sensor nodes, assuming that the network can tolerate $f_{\max }$ nodes, so the voting system can be used to study the reliability of subnets. The reliability of different operation status of network is shown in the following:

(1) $n$ nodes work normally. The network reliability is:

$R_{1}(t)=\left[R_{0}(t)\right]^{n}$

(2) 1 node has faults and $n-1$ nodes work normally. The network reliability is:

$R_{2}(t)=\mathrm{C}_{n}^{n-1}\left[R_{0}(t)\right]^{n-1}\left[1-R_{0}(t)\right]$

(3) 2 nodes have faults and $n$-2 nodes work normally. The network reliability is:

$R_{3}(t)=\mathrm{C}_{n}^{n-2}\left[R_{0}(t)\right]^{n-2}\left[1-R_{0}(t)\right]^{2}$

(4) $f_{\max }$ nodes have faults and $n-f_{\max }$ nodes work normally. The network reliability is:

$R_{j}(t)=\mathrm{C}_{n}^{n-f_{\max }}\left[R_{0}(t)\right]^{n-f_{\max }}\left[1-R_{0}(t)\right]^{f_{\max }}$

(5) $f_{\max }+1$ nodes have faults, the network cannot work normally. The network reliability is: $R_{j+1}=0$.

Therefore, when the network can tolerate $f_{\max }$ nodes, the reliability of each subnet is:

$R_{\mathrm{c}}(t)=\sum_{i=1}^{j} R_{i}(t)=\sum_{i=n-f_{\max }}^{n} \mathrm{C}_{n}^{i}\left[R_{0}(t)\right]^{i}\left[1-R_{0}(t)\right]^{n-i}$

For the WSN-MSWT composed of $m$ subnets, subnets form the star topology network. To let the entire network operate normally, each subnet that works alone should operate without faults. This paper uses the voting system to study the reliability model. The reliability model of the voting system can be presented in the following: 
Table 2. Deployment of sensors in WSN-MSWT.

\begin{tabular}{|c|c|c|c|c|}
\hline Network parameters & Subnets $m$ & Subnet nodes $n$ & Fault-tolerated nodes $f_{\max }$ & Total nodes $k$ \\
\hline Mode 1 & 8 & 6 & 16 & 48 \\
\hline Mode 2 & 12 & 6 & 24 & 72 \\
\hline Mode 3 & 8 & 12 & 32 & 72 \\
\hline Mode 4 & 4 & 12 & 16 & 48 \\
\hline
\end{tabular}

Table 3. Time of different reliability in different mode. (unit: $10^{4} \mathbf{h}$ ).

\begin{tabular}{|c|c|c|c|c|c|}
\hline Reliability & $\mathbf{0 . 9 5}$ & $\mathbf{0 . 9 0}$ & $\mathbf{0 . 8 0}$ & $\mathbf{0 . 5 0}$ & 20.6 \\
\hline \hline Mode 1 & 7.5 & 9.7 & 13 & 17.4 & 35.2 \\
\hline Mode 2 & 6.4 & 8.4 & 11.1 & 23.2 & 29.2 \\
\hline Mode 3 & 11.5 & 13.8 & 16.8 & 28.8 & 44.2 \\
\hline Mode 4 & 13.7 & 16.6 & 25.9 & 4.1 \\
\hline
\end{tabular}

$R(t)=\left[R_{\mathrm{c}}(t)\right]^{m}$

\section{SIMULATION AND ANALYSIS}

In this paper, amounts of subnets, nodes of subnets, system fault-tolerated nodes and system nodes are considered to be the network parameters. Additionally, the system reliability and the system working time are regarded as evaluation indexes.

\subsection{Reliability of WSN-MSWT}

The network parameters of WSN-MSWT are shown in Table 2. In Table 2, there are four kinds of nodes deployments. The reliability function $R(t)$ can be obtained by equations (22) and (23)( Fig. (3)). Meanwhile, the time $t$ can be calculated as shown in Table 3.

In Fig. (3), the curve of reliability function of the monitoring system have three sections. In the first section, the reliability is very high with $R(t)>0.9 . R(t)$ decreases slowly as the time increases. In the second section, $R(t)$ decreases rapidly as the time increases with $0.1<R(t)<0.9$. In the third section, the reliability is very slow with $R(t)<0.1 . R(t)$ is approaching 0 and the speed of decreasing becomes slower again.

Comparing mode 1 with mode 2 , when the amounts of nodes in each subnet and fault-tolerated nodes are unchanged, the system reliability decreases obviously as the amount of subnets increases. Therefore, the increasing amount of subnets in the monitoring system reduces the monitoring system reliability.

Comparing mode 1 with mode 3 , when the amount of subnets keeps unchanged and the amounts of nodes in subnets and fault-tolerated nodes increase, the system reliability improves. However, the reliability decreasing speed accelerates. Therefore, if the subnet amount is unchanged for the transmission system, increasing the nodes of subnet will improve the monitoring system reliability.

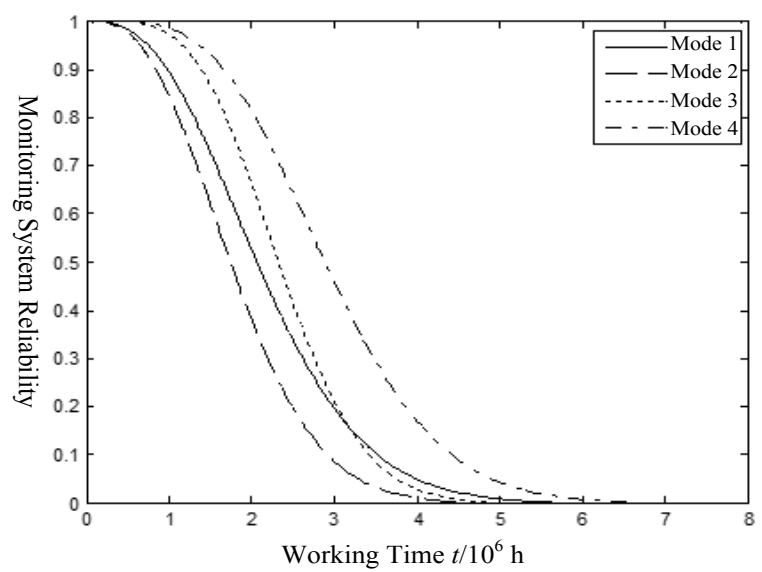

Fig. (3). Reliability function $R(t)$ in different mode.

Comparing mode 1 with mode 4 , when the amounts of the system nodes and fault-tolerated nodes are unchanged, reducing appropriately subnet amount and increasing nodes amount of each subnet can significantly improve the system reliability.

\subsection{Working Time of Monitoring Network}

According to the reliability evaluation model of monitoring system, then normal working time of system can be calculated.

Take $\lambda(t)=\lambda=10^{-6}$ and $R(0)=1$ combining with Equation (23), Equation (24) is obtained as follows: 


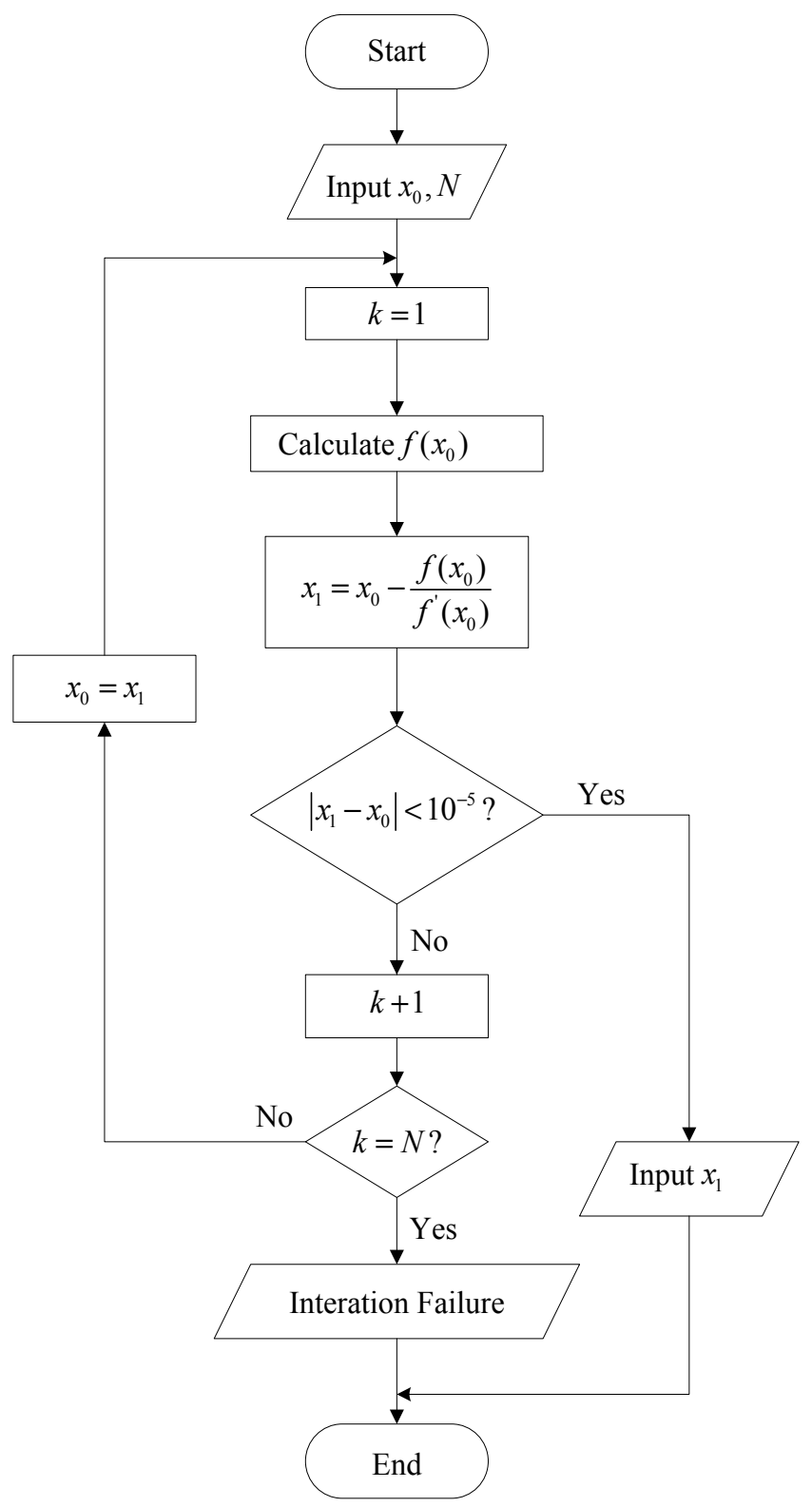

Fig. (4). Flow chart of newton iterative method.

Table 4. System normal working hours of different reliability in different mode.

\begin{tabular}{|c|c|c|c|c|}
\hline Network Parameters & Mode 1 & Mode 2 & Mode 3 & Mode 4 \\
\hline \hline System normal working time (hour) & 93758 & 73926 & 152610 & 197580 \\
\hline System normal working time (year) & 10.7 & 8.4 & 17.4 & 22.6 \\
\hline
\end{tabular}

$10^{-6} t=-\ln [R(t)]^{m}$

Since Equation (24) is a transcendental equation, use Newton iterative method to solve Equation (24).

Let $f(t)=10^{-6} t+\ln [R(t)]^{m}$, the Newton iteration method is shown in Fig. (4).

As shown in Table 4, the normal working time of different mode has the same trend with the time in Fig. (3). For further studying of influence of system network parameters on system normal working hours, use mode 1 as an example. There are two kinds of situations:

(1) $k$ and $f_{\text {max }}$ keep constant, and $n$ changes

The curve of the normal working time with changing time is as shown in Fig. (5). It can be seen that system working time increases as amount of subnet nodes increases. That is because the network of monitoring system adopts the mixture of star and mesh topology, wherein the each inner subnet uses mesh topology while the network between subnets 


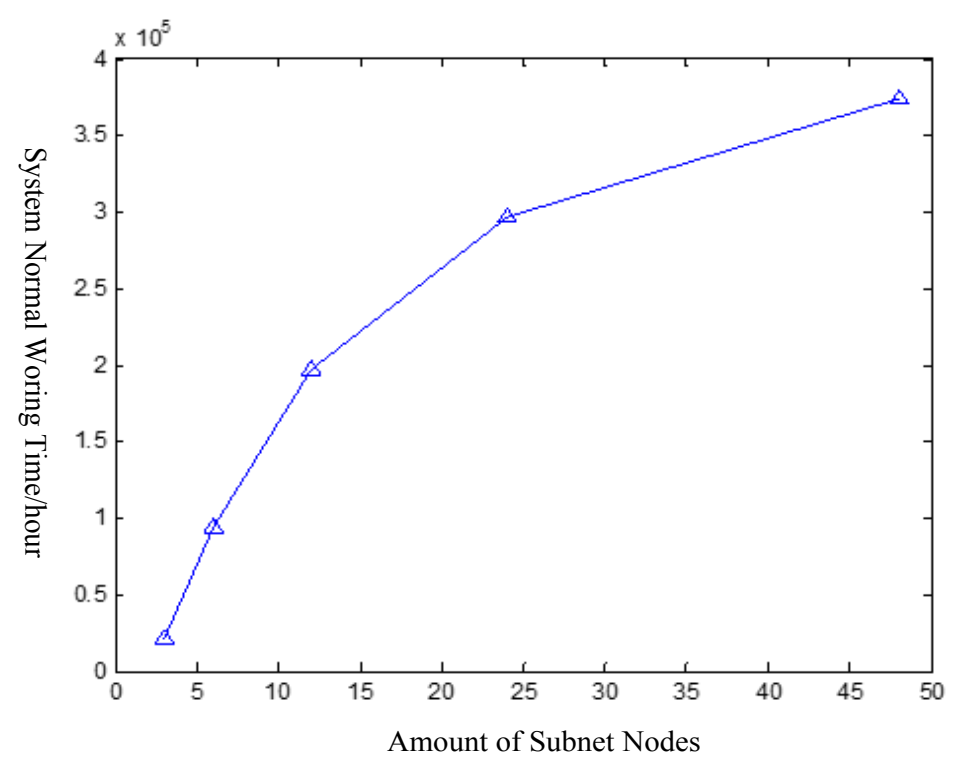

Fig. (5). System normal working hours changing with $n$.

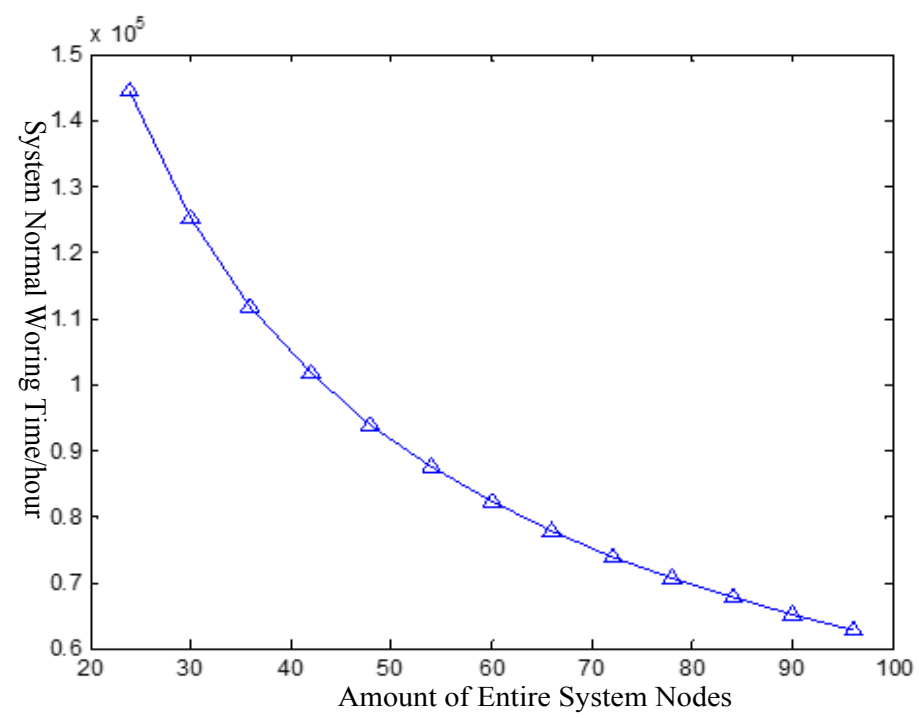

Fig. (6). System normal working hours changing with $k$.

and base station uses star topology. As nodes amount of system does not change, subnet amount decreases while amount of each subnet nodes increases. Then the mesh topology structure with strong reliability increases, the star topology structure with weak reliability decreases. Thus, the system normal working time increases as $n$ increases. In the extreme case, when $n=48$ and $m=1$, the system normal working time reaches the maximum because the entire system network has mesh topology.

(2) $n$ and amount of fault-tolerated nodes in subnet keep constant, and $\mathrm{k}$ changes

The curve of the normal working time with changing $\mathrm{k}$ is as shown in Fig. (6). In conclusion, the system working time decreases as amount of system nodes increases. The amounts of subnet nodes and fault-tolerated nodes keep unchanged, so the reliability of each subnet does not change. However, increasing $k$ means increasing subnets amount. As a result, the system reliability becomes weaker and system normal working time decreases as more subnets in the entire system form the star topology structure.

\section{CONCLUSION}

This paper proposes the hierarchical network topology of WSN-based condition monitoring system for wind turbine, and studies the reliability modeling and analysis. The simulation results show the following conclusion: 
1) When the amounts of each subnet nodes and faulttolerated nodes are unchanged, increasing the subnet amount reduces the reliability of monitoring system.

2) For monitoring the important transmission system, if the subnet amount keeps unchanged, the nodes amount of subnet will increase. Then, the system reliability can be improved.

3) When the system nodes amount and the fault-tolerated nodes keep unchanged, reducing appropriately the subnets amount means improving the system reliability.

\section{CONFLICT OF INTEREST}

The authors confirm that this article content has no conflicts of interest.

\section{ACKNOWLEDGEMENTS}

This work is supported by the National Natural Science Foundation of China (no.51307044), the Natural Science Foundation of Jiangsu Province of China (no.BK2012409), Six Talent Peaks Project of Jiangsu Province of China(no.2014-XNY-008) and the Project of China Three Gorges New Energy Corporation.

\section{REFERENCES}

[1] C.X. Wang, Z.X. Lu and Y. Qiao, "A Consideration of the Wind Power Benefits in Day-Ahead Scheduling of Wind-Coal Intensive Power Systems," IEEE Transactions on Power Systems, vol. 28, no. 1, pp. 236-245, 2013.

[2] Z.X. Fu, and Y Yue, "Status and Prospects on Condition Monitoring Technologies of Offshore Wind Turbine," Automation of Electric Power System, vol. 36, no. 21, pp. 121129,2012

[3] W.X. Yang, P.J. Tavner, and et al, "Cost-Effective Condition Monitoring for Wind Turbines," IEEE Transactions on Industrial Electronics, vol. 57, no. 1, pp. 263-271, 2010.

[4] J.A.R. Azevedo, and F.E.S. Santos, "Energy Harvesting from Wind and Water for Autonomous Wireless Sensor Nodes," IET Circuits, Devices \& Systems, vol. 6, no. 6, pp. 413-420, 2012.
[5] Z.X. Fu, M. Zhao,Y. Yuan, and et al, "Construction Strategies for Offshore Wind Turbine Condition Monitoring System Based on Wireless Sensor Networks," Automation of Electric Power System, vol. 38, no. 7, pp. 23-28, 2014.

[6] C. Popeanga, R. Dobrescu, and N. Cristove, "Smart Monitoring and Controlling of Wind Turbines Farms Based on Wireless Sensors Networks", 2012 1st International Conference on Systems and Computer Science (ICSCS), pp. 1-6, 2012.

[7] C.L. Hus, and T.Y. Hsu, "The Practical Design of Constructing Data Transition Interface with ZigBee WSN-Example with Small Scaled Wind-Power Electricity Generator System," Journal of Software, vol. 3, no. 8, pp. 49-56, 2008.

[8] Y.K. Tan, and S.K. Panda, "Self-autonomous Wireless Sensor Nodes with Wind Energy Harvesting for Remote Sensing of Wind-driven Wildfire Spread," IEEE Transactions on Instrumentation and Measurement, vol. 60, no. 4, pp. 1367-1377, 2011.

[9] A.D. Spacek, O.H. Ando Junior, J.M. Neto, and et al, "Management of Mechanical Vibration and Temperature in Small Wind Turbines Using Zigbee Wireless Network," IEEE Latin America Transactions, vol. 11, no. 1, pp. 512-517, 2013.

[10] C. Liu, S.Y. Zhang, H.B. Feng, and et al, "The Research on Wireless Sensors Network Reliability," Proceedings of 2008 International conference on Wireless Communications, Networking and Mobile Computing, pp. 1-4, 2008.

[11] A.A. Aziz, Y.A. Sekercioglu, P. Fitzpatrick, and et al, "A Survey on Distributed Topology Control Techniques for Extending The Lifetime of Battery Powered Wireless Sensor Networks," IEEE Communications Surveys \& Tutorials, vol. 15, no. 1, pp. 121144, 2013.

[12] C. Vasar, O. Prostean, I. Filip, and et al, "A Reliability Analysis for Wireless Sensor Networks in a Wind Farm," XXII International Symposium on Information, Communication and Automation Technologies, pp. 1-5, 2009.

[13] M. Zorzi, and R.R. Rao. "Geographic Random Forwarding (GeRaF) for Ad Hoc and Sensor Networks: Multi-Hop Performance," IEEE Transactions on Mobile Computing, vol. 2, no. 3 .

[14] I.F. Akyildiz, W. Su, Y. Sankarasubramaniam, and et al, "Wireless Sensor Network: A Survey," Computer Networks, vol. 38, pp. 393-422, 2002.

[15] P. Caselitz, and J. Giebhargt, "Rotor Condition Monitoring for Improved Operational Safety of Offshore Wind Energy Converters," ASME Transactions, Journal of Solar Energy Engineering, vol. 127, pp. 253-261, 2005.

[16] P.E. Labeau, and C. Smidts, "Dynamic Reliability: Towards an Integrated Platform for Probabilistic Risk Assessment," Reliability Engineering and System Safety, vol. 68, pp. 219-254, 2000.

Received: September 16, 2014

Revised: December 23, 2014

Accepted: December 31, 2014

(C) Zhixin et al.; Licensee Bentham Open.

This is an open access article licensed under the terms of the Creative Commons Attribution Non-Commercial License (http://creativecommons.org/licenses/by-nc/3.0/) which permits unrestricted, non-commercial use, distribution and reproduction in any medium, provided the work is properly cited. 